too late in the course of the disease. Further analyses are needed to define more appropriate selection criteria for heart teams in choosing a percutaneous strategy in patients with secondary mitral regurgitation.

Daniel Grinberg, M.D.

Jean-François Obadia, M.D., Ph.D.

Hôpital Louis Pradel

Lyon, France

jean-francois.obadia@chu-lyon.fr

Bernard lung, M.D., Ph.D.

Hôpital Bichat

Paris, France

Dr. Grinberg reports no potential conflict of interest relevant to this letter. Since publication of their article, Drs. Obadia and Iung report no further potential conflict of interest.
1. Stone GW, Lindenfeld J, Abraham WT, et al. Transcatheter mitral-valve repair in patients with heart failure. $\mathrm{N}$ Engl J Med 2018;379:2307-18.

2. Nishimura RA, Otto CM, Bonow RO, et al. 2017 AHA/ACC focused update of the 2014 AHA/ACC guideline for the management of patients with valvular heart disease: a report of the American College of Cardiology/American Heart Association Task Force on Clinical Practice Guidelines. Circulation 2017; 135(25):e1159-e1195.

3. Baumgartner H, Falk V, Bax JJ, et al. 2017 ESC/EACTS Guidelines for the management of valvular heart disease. Eur Heart J 2017;38:2739-91.

4. Grinberg D, Donal E, Obadia J-F. What do MITRA-FR and COAPT teach us about the percutaneous treatment of secondary mitral regurgitation? EuroIntervention 2019;14:1713-5 (https:// www.pcronline.com/eurointervention/150th_issue/volume-14/ number-17/295/what-do-mitra-fr-and-coapt-teach-us-about-the -percutaneous-treatment-of-secondary-mitral-regurgitation.html). DOI: 10.1056/NEJMc1903624

\title{
Transcatheter Mitral-Valve Repair in Patients with Heart Failure
}

TO THE EDITOR: The trials conducted by Obadia et al. (Percutaneous Repair with the MitraClip Device for Severe Functional/Secondary Mitral Regurgitation [MITRA-FR] $)^{1}$ and Stone et al. (Cardiovascular Outcomes Assessment of the MitraClip Percutaneous Therapy for Heart Failure Patients with Functional Mitral Regurgitation [COAPT] $)^{2}$ (both in the Dec. 13 issue) provide contradictory evidence on the efficacy of transcatheter mitral-valve repair for functional mitral regurgitation. Although the reasons for the differences in outcomes are unclear, inconsistencies in the data in the COAPT trial highlight the difficulty of quantifying the severity of functional mitral regurgitation. Patients had a mean $( \pm S D)$ effective regurgitant orifice area of $0.41 \pm 0.15 \mathrm{~cm}^{2}$, which would correspond to a mitral regurgitant

\begin{tabular}{|c|c|c|}
\hline Characteristic & Device Group & Control Group \\
\hline LVEDV - ml & $194.4 \pm 69.2$ & $191.0 \pm 72.9$ \\
\hline LVESV - ml & $135.5 \pm 56.1$ & $134.3 \pm 60.3$ \\
\hline Effective regurgitant orifice area $-\mathrm{cm}^{2}$ & $0.41 \pm 0.15$ & $0.40 \pm 0.15$ \\
\hline $\begin{array}{l}\text { Total stroke volume (LVEDV minus LVESV) } \\
\quad-\mathrm{ml}\end{array}$ & 59.4 & 56.7 \\
\hline Mitral regurgitant volume $-\mathrm{ml} \dagger$ & $>45-60$ & $>45-60$ \\
\hline $\begin{array}{l}\text { Forward stroke volume (total stroke volume } \\
\text { minus mitral regurgitant volume) }-\mathrm{ml}\end{array}$ & $0-15$ & $0-15$ \\
\hline
\end{tabular}

* Plus-minus values are means \pm SD. LVEDV denotes left ventricular end diastolic volume, and LVESV left ventricular end systolic volume.

$\uparrow$ The estimated mitral-regurgitant volume is based on an effective regurgitant orifice area of $0.40 \mathrm{~cm}^{2}$. volume of at least 45 to $60 \mathrm{ml}^{3}$ Data from the trial also indicate that patients had a total left ventricular stroke volume of approximately $60 \mathrm{ml}$ (left ventricular end diastolic volumeminus left ventricular end systolic volume) (Table 1). These two findings are incompatible. If the total ventricular stroke volume is $60 \mathrm{ml}$, then the regurgitant volume is a portion of this and the forward stroke volume is the balance (total stroke volume equals mitral regurgitant volume plus forward stroke volume). Therefore, in the COAPT trial, the forward stroke volume is 0 to $15 \mathrm{ml}$, which would be incompatible with life. These data require clarification.

Juan A. Crestanello, M.D.

Jae K. Oh, M.D.

Hartzell V. Schaff, M.D.

Mayo Clinic

Rochester, MN

crestanello.juan@mayo.edu

No potential conflict of interest relevant to this letter was reported.

1. Obadia J-F, Messika-Zeitoun D, Leurent G, et al. Percutaneous repair or medical treatment for secondary mitral regurgitation. N Engl J Med 2018;379:2297-306.

2. Stone GW, Lindenfeld JA, Abraham WT, et al. Transcatheter mitral-valve repair in patients with heart failure. $\mathrm{N}$ Engl J Med 2018;379:2307-18

3. Enriquez-Sarano M, Seward JB, Bailey KR, Tajik AJ. Effective regurgitant orifice area: a noninvasive Doppler development of an old hemodynamic concept. J Am Coll Cardiol 1994;23:443-51.

DOI: 10.1056/NEJMc1903982

TO THE EDITOR: The COAPT trial confirms that mitral-valve repair for severe regurgitation decreases the morbidity and mortality associated 
with secondary mitral-valve disease. The trial also demonstrates the essential role of imaging in planning and guiding intervention.

In the trial, anatomical lesions that resulted in regurgitation were identified with transesophageal echocardiography during pretrial evaluation. Trial inclusion required an interventionalist to select patients whose anatomy was suitable for percutaneous repair. This requirement introduced a strong selection bias in favor of longterm benefit with percutaneous intervention. In contrast, image-guided anatomical selection was not used in a related trial conducted by the Cardiothoracic Surgical Trials Network. ${ }^{1}$ This disparity may account for the difference in results between these two trials. Lack of standardized preoperative image guidance may also be contributing to the trend away from surgical repair of primary mitral-valve disease. ${ }^{2}$ In contrast, protocol-driven, image-guided surgical repair is associated with excellent freedom from recurrence of both primary and secondary regurgitation. ${ }^{3}$

Durable mitral-valve repair should be performed in patients with severe regurgitation. Anatomically based image guidance is mandatory for both percutaneous and surgical repair. Trial design should include indexes of anatomical distortion. ${ }^{4}$ We believe that evidence-based anatomical staging of secondary mitral-valve disease should be part of any repair.

Daniel H. Drake, M.D.

Munson Medical Center

Traverse City, MI

daniel.h.drake@gmail.com

Karen G. Zimmerman, B.S., A.C.S.

West Virginia University Heart and Vascular Institute Morgantown, WV

David A. Sidebotham, M.B., Ch.B.

Auckland City Hospital

Auckland, New Zealand

No potential conflict of interest relevant to this letter was reported.

1. Acker MA, Parides MK, Perrault LP, et al. Mitral-valve repair versus replacement for severe ischemic mitral regurgitation. $\mathrm{N}$ Engl J Med 2014;370:23-32.

2. Gammie JS, Chikwe J, Badhwar V, et al. Isolated mitral valve surgery: the Society of Thoracic Surgeons Adult Cardiac Surgery Database analysis. Ann Thorac Surg 2018;106:716-27.

3. Drake DH, Zimmerman KG, Hepner AM, Nichols CD. Echoguided mitral repair. Circ Cardiovasc Imaging 2014;7:132-41.

4. Drake DH, Zimmerman KG, Sidebotham DA. Transesophageal echocardiography for surgical repair of mitral regurgitation. In: Otto CM, ed. The practice of clinical echocardiography. 5th ed. Philadelphia: Elsevier, 2016:343-67.

DOI: 10.1056/NEJMc1903982
TO THE EDITOR: Transcatheter repair of secondary mitral regurgitation was associated with improved survival and symptomatic benefit in the COAPT trial. No beneficial outcome was reported in the MITRA-FR trial.

Secondary mitral regurgitation responds to medical treatment of heart failure as long as heart failure drives secondary mitral regurgitation. However, when the roles reverse and secondary mitral regurgitation drives heart failure, the resulting entity - valvular heart failure no longer responds to medical treatment. ${ }^{1}$ The severity of mitral regurgitation was greater, and left ventricular dilatation less severe, in the COAPT trial population, suggesting that mitral regurgitation was an important contributor to heart failure symptoms. Could the COAPT trial results be due to the presence of valvular heart failure, and would mitral-valve replacement have produced similar results?

Left atrial pressure reduction with an interatrial shunt device ameliorates symptoms in heart failure with preserved ejection fraction. ${ }^{2}$ Furthermore, left atrial pressure reduction with transcatheter repair of mitral regurgitation is associated with improved exercise tolerance in patients with primary mitral regurgitation and in COAPT trial candidates. ${ }^{3}$ Could left atrial pressure reduction explain the COAPT trial results?

Madalina Garbi, M.D., M.A.

King's College London

London, United Kingdom

mgarbi@doctors.org.uk

Patrizio Lancellotti, M.D., Ph.D.

University of Liege Hospital

Liege, Belgium

No potential conflict of interest relevant to this letter was reported.

1. Lancellotti P, Garbi M. Progression of secondary mitral regurgitation: from heart failure to valvular heart failure. Eur Heart J Cardiovasc Imaging 2018;19:613-4.

2. Kaye DM, Hasenfuß G, Neuzil P, et al. One-year outcomes after transcatheter insertion of an interatrial shunt device for the management of heart failure with preserved ejection fraction. Circ Heart Fail 2016;9(12):e003662.

3. Maor E, Raphael CE, Panaich SS, et al. Acute changes in left atrial pressure after MitraClip are associated with improvement in 6-minute walk distance. Circ Cardiovasc Interv 2017;10(4): e004856.

DOI: 10.1056/NEJMc1903982

TO THE EDITOR: Many groups are trying to reconcile the divergence in results reported in the MITRA-FR and COAPT trials regarding the effectiveness of transcatheter mitral-valve repair in patients with secondary mitral regurgitation. 
Why is there an absence of benefit in the MITRA-FR trial and a dramatic improvement in clinical outcomes in the COAPT trial? The left ventricular dimension was, on average, greater among patients in the MITRA-FR trial, which reflects more advanced disease. Perhaps more important, in the COAPT trial, nearly $60 \%$ of screened patients (911 of 1576) did not undergo randomization. The success of the MitraClip device is critically dependent on whether strict echocardiographic criteria are met. Enrollment was supervised by the sponsor (Abbott) in the COAPT trial and was physician-led and independent of industry in the MITRA-FR trial. The results of the COAPT trial suggest that transcatheter mitral-valve repair may be beneficial in highly selected patients with heart failure who have secondary mitral regurgitation. However, we are concerned that the real-world effect of this therapy may prove to be more like the results presented in the MITRA-FR trial.

Dimitri Kalavrouziotis, M.D.

Pierre Voisine, M.D.

Siamak Mohammadi, M.D.

Quebec Heart and Lung Institute

Quebec City, QC, Canada

dimitri.kalavrouziotis@criucpq.ulaval.ca

No potential conflict of interest relevant to this letter was reported.

DOI: 10.1056/NEJMc1903982

THE AUTHORS REPLY: Crestanello et al. question the apparently low forward stroke volume calculated from the total left ventricular stroke volume, which was determined by applying Simpson's method to two-dimensional biplane echocardiographic measurements and using an assumed regurgitant volume. The actual mean forward stroke volume in the COAPT trial as measured with Doppler was $51 \mathrm{ml}$, and the regurgitant volume as measured with the use of the PISA (proximal isovelocity surface area) method was $59 \mathrm{ml}$, values that are consistent with severe mitral regurgitation. There are several reasons for the discrepancies from Crestanello's theoretical extrapolation, in which two-dimensional and Doppler data are combined, the most important being the substantial underestimation of left ventricular volume (and stroke volume), as determined by two-dimensional echocardiography with the use of Simpson's rule, especially in patients with dilated ventricles, such as those enrolled in the COAPT trial. ${ }^{1}$

Drake et al. posit that a lack of imaging guidance resulted in a high rate of recurrence of mitral regurgitation after surgical repair with a downsized annuloplasty ring in the trial conducted by the Cardiothoracic Surgical Trials Network. That trial used detailed echocardiographic analysis to gauge patient suitability for inclusion. A post hoc subanalysis defined the anatomical features shown on echocardiography that were predictive of a durable surgical repair. ${ }^{2}$ Ongoing, detailed echocardiographic analyses in the COAPT trial will further delineate the anatomical features that predict favorable outcomes after transcatheter mitral-leaflet approximation. We do agree that image-guided assessment is essential to the identification of a responder population and that in the future it may direct patientspecific intervention (leaflet repair, annuloplasty, or valve replacement).

What Garbi and Lancellotti term valvular heart failure secondary to mitral regurgitation, Grayburn et al. ${ }^{3}$ designate as disproportionate mitral regurgitation and Carabello ${ }^{4}$ calls tertiary mitral regurgitation. Regardless of the nomenclature, we agree that relative to the MITRA-FR trial, the COAPT trial investigators enrolled a greater proportion of patients in whom prognosis was dictated more by the severity of mitral regurgitation than by the degree of left ventricular dysfunction - a major reason why patients in the COAPT trial, but not those in the MITRA-FR trial, benefited from transcatheter mitral-valve repair. We further agree that reduction in left atrial pressure (and volume) was probably responsible for many of the clinical benefits associated with transcatheter mitral-valve repair in the COAPT trial. Mitral-valve replacement offers the potential for greater reduction in mitral regurgitation than transcatheter mitral-valve repair. Whether the procedure will provide sufficiently greater clinical effectiveness warranting a potentially more complex and complicated procedure is uncertain and can only be addressed by means of adequately powered randomized trials.

Finally, Kalavrouziotis et al. are incorrect in stating that enrollment in the COAPT trial was "supervised" by the sponsor. Rather, investigators at each site determined whether screened patients met prespecified enrollment criteria, a determination that was then confirmed by a physicianled, sponsor-independent, central eligibility committee and echocardiographic core laboratory. We believe that practitioner fidelity to the inclusion and exclusion criteria used in the COAPT trial should lead to duplication of our results in the real world. 
Gregg W. Stone, M.D.

Columbia University Medical Center

New York, NY

gs2184@columbia.edu

Neil J. Weissman, M.D.

MedStar Health Research Institute

Hyattsville, MD

Michael J. Mack, M.D.

Baylor Scott \& White Heart Hospital Plano

Plano, TX

\section{for the COAPT Investigators}

Since publication of his article, Dr. Stone reports receiving consulting fees from Abiomed, Ancora, and Backbeat and having equity in Applied Therapeutics. No further potential conflict of interest relevant to this letter was reported.
1. Dorosz JL, Lezotte DC, Weitzenkamp DA, Allen LA, Salcedo EE. Performance of 3-dimensional echocardiography in measuring left ventricular volumes and ejection fraction: a systematic review and meta-analysis. J Am Coll Cardiol 2012;59:1799-808. 2. Kron IL, Hung J, Overbey JR, et al. Predicting recurrent mitral regurgitation after mitral valve repair for severe ischemic mitral regurgitation. J Thorac Cardiovasc Surg 2015;149(3):75261.e1.

3. Grayburn PA, Sannino A, Packer M. Proportionate and disproportionate functional mitral regurgitation: a new conceptual framework that reconciles the results of the MITRA-FR and COAPT trials. JACC Cardiovasc Imaging 2019;12:353-62.

4. Carabello BA. MitraClip and tertiary mitral regurgitation mitral regurgitation gets curiouser and curiouser. JAMA Cardiol 2019 March 6 (Epub ahead of print).

DOI: 10.1056/NEJMc1903982

\section{Organoids - Preclinical Models of Human Disease}

TO THE EDITOR: Li and Izpisua Belmonte (Feb. 7 issue $)^{1}$ systematically used organoids to establish various kinds of preclinical models of human diseases. However, they do not fully discuss possible modeling of the tumor immune microenvironment.

We do not entirely agree with the authors that three-dimensional organoids are more limited than animal models because of their lack of vascularization and an immune system. Increasing numbers of studies have supported the use of three-dimensional organoid models for holistic study of the tumor immune microenvironment. ${ }^{2-5}$ Three-dimensional organoids represent the in vivo interaction of tumor and immune cells in the tumor microenvironment, and patientderived organoids incorporating tumor stromal components may help to promote the development of precision therapies for cancer. ${ }^{2}$

Moreover, human tumor suspension-derived microspheroids containing immune cells can have a response to immunotherapeutic agents and provide support for the use of three-dimensional organoid models to evaluate changes in the tumor immune microenvironment. ${ }^{3,4}$ Although organoids may be a new platform for preclinical models of human diseases, their use for analyzing the tumor immune microenvironment and tumor immunotherapy should be carefully considered.

Chuanli Ren, M.D.

Chongxu Han, M.D.

Clinical Medical College of Yangzhou University

Yangzhou, China

renchl@163.com

No potential conflict of interest relevant to this letter was reported.
1. Li M, Izpisua Belmonte JC. Organoids - preclinical models of human disease. N Engl J Med 2019;380:569-79.

2. Neal JT, Li X, Zhu J, et al. Organoid modeling of the tumor immune microenvironment. Cell 2018;175(7):1972-1988.e16.

3. Jenkins RW, Aref AR, Lizotte PH, et al. Ex vivo profiling of PD-1 blockade using organotypic tumor spheroids. Cancer Discov 2018;8:196-215.

4. Deng J, Wang ES, Jenkins RW, et al. CDK4/6 inhibition augments antitumor immunity by enhancing T-cell activation. Cancer Discov 2018;8:216-33.

5. Dijkstra KK, Cattaneo CM, Weeber F, et al. Generation of tumor-reactive T cells by co-culture of peripheral blood lymphocytes and tumor organoids. Cell 2018;174(6):1586-1598.e12.

DOI: 10.1056/NEJMc1903253

TO THE EDITOR: Li and Izpisua Belmonte comprehensively review the translational potential of organoid technology, including the ability to create microtissues with the use of scaffold-free synthesis, bioprinted scaffolds, or biopolymers. Similarly, three-dimensional printing techniques include additive manufacturing of unmodified as well as bioactivated materials; these techniques enable organoid studies of complex microenvironments that may be more clinically relevant. ${ }^{1,2}$ Threedimensional printed templates can be used to construct tailored organoid systems such as organoid "cages," cell exclusion spacers, multicellular invasion systems, and high-throughput cell-culture wells that incorporate bioactive agents. ${ }^{2-4}$ Merging such three-dimensional printed design elements into trials of organoids would permit exceptionally diverse biomimetic cellular interactions and more uniform localized drugrelease systems for organoid pharmacodynamic models.

Such constructs are easy to customize and can be produced with commercial three-dimensional printers using preexisting design tem- 